\title{
Disrupting Crystal Growth Through Molecular Recognition: Designer Therapies for Kidney Stone Prevention
}

\author{
Alexander G. Shtukenberg, ${ }_{1}^{1}$ Longqin Hu, ${ }^{2}$ Amrik Sahota, ${ }^{3}$ Bart Kahr ${ }^{1}$ and Michael D. Ward ${ }^{1, *}$ \\ ${ }^{1}$ Department of Chemistry and Molecular Design Institute, New York University, 100 Washington Square East, New \\ York, NJ, 10003-6688. ${ }^{2}$ Department of Medicinal Chemistry, Ernest Mario School of Pharmacy, Rutgers University, \\ Piscataway, NJ 08854. ${ }^{3}$ Department of Genetics, Life Sciences Building, Rutgers University, Piscataway, NJ 08854. \\ Corresponding author*Email: mdw3@nyu.edu
}

\section{Supporting Information: Video Log}

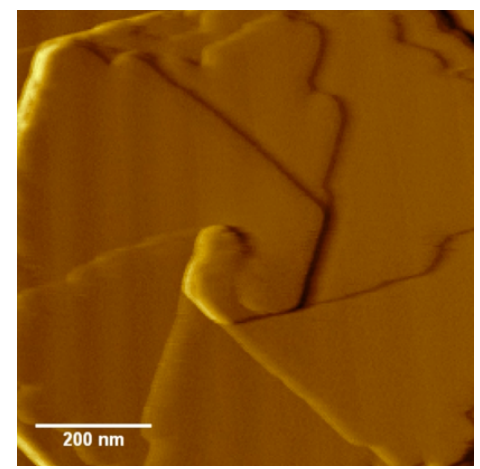

Video S1. Real-time in situ AFM video of a growth hillock on the L-cystine (0001) surface generated by a single dislocation, near the dislocation core. Reproduced with permission from reference 1. Copyright 2013 United States National Academy of Sciences.
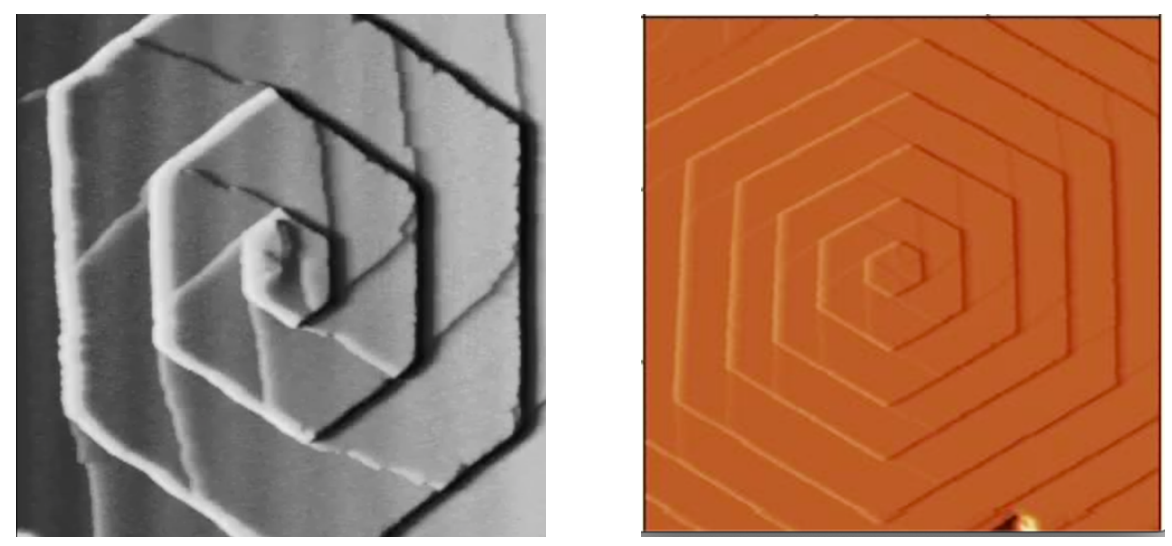

Video S2. Real-time in situ AFM videos of a growth hillock on the L-cystine (0001) surface generated by a single dislocation (A, left; $B$, right). The images give the appearance of stacked islands, but the micromorphology is the result of growing interlaced anisotropic spirals that bunch into unit cell-high steps, each bunch generated on top of the previous one below. Islands near the top are smaller because they emerged after the ones below. (left) Reproduced with permission from reference 2. Copyright 2010 American Association for the Advancement of Science (United States). (right) Reproduced with permission from reference 1. Copyright 2013 United States National Academy of Sciences. 


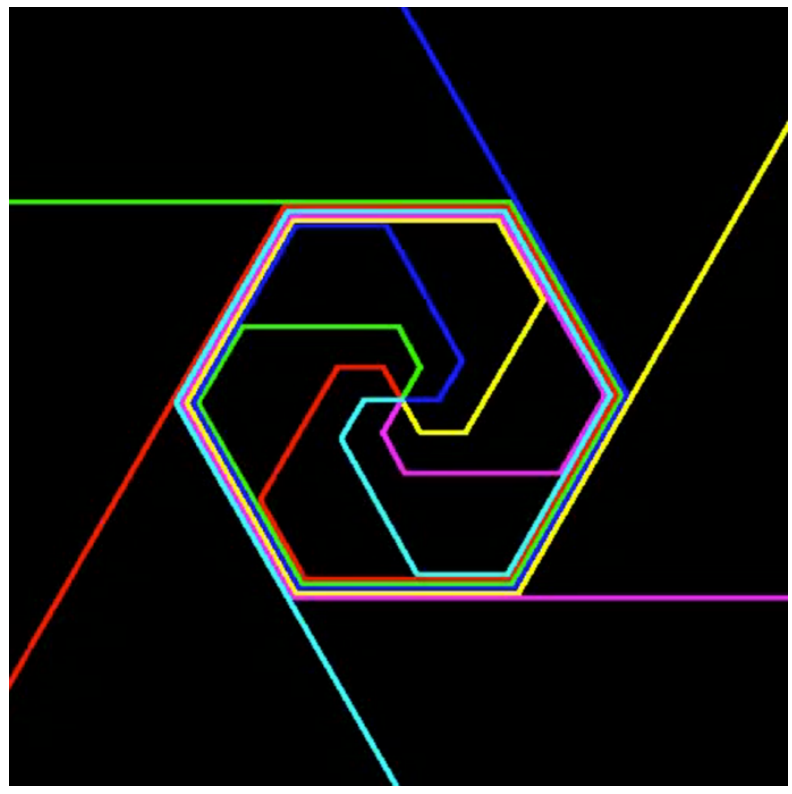

Video S3. Simulation of a growth hillocks on the L-cystine (0001) surface generated by a single dislocation, using step velocity data obtained from the AFM measurements of the step velocities along the six different directions in a single elementary $c / 6$ layer. The simulation mirrors the L-cystine (0001) morphology observed in Video S2 and Figure 4, but it reveals that islands are a consequence of interlaced spirals. The six different colors correspond to the six different elementary layers in the unit cell along the $c$-axis. Reproduced with permission from reference 1. Copyright 2013 United States National Academy of Sciences.

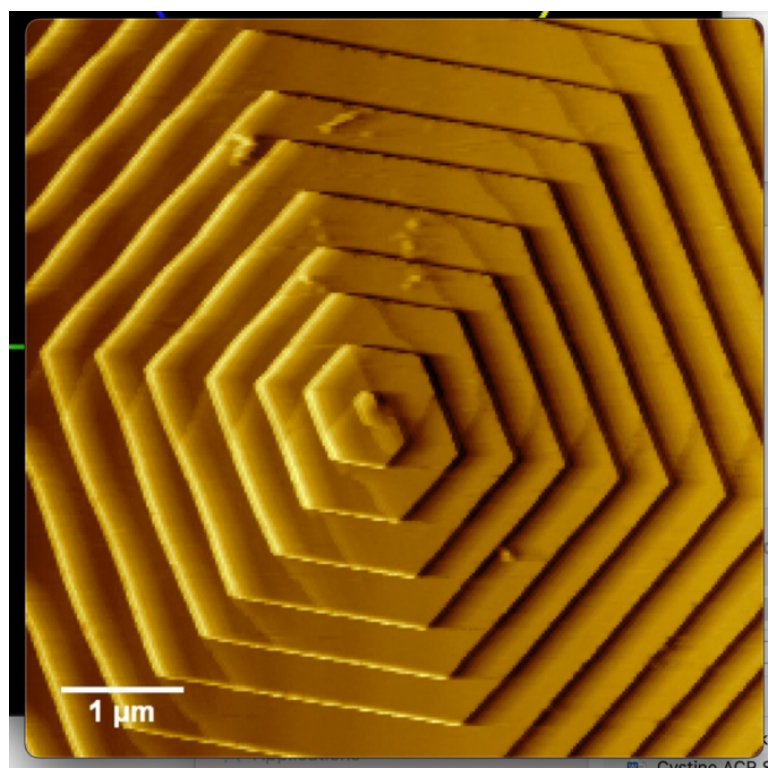

Video S4. Real-time in situ AFM of a macrospiral formed on the L-cystine (0001) surface generated by a Frank-Read source comprising a closely spaced pair of heterochiral dislocations. Reproduced with permission from reference 1. Copyright 2013 United States National Academy of Sciences. 


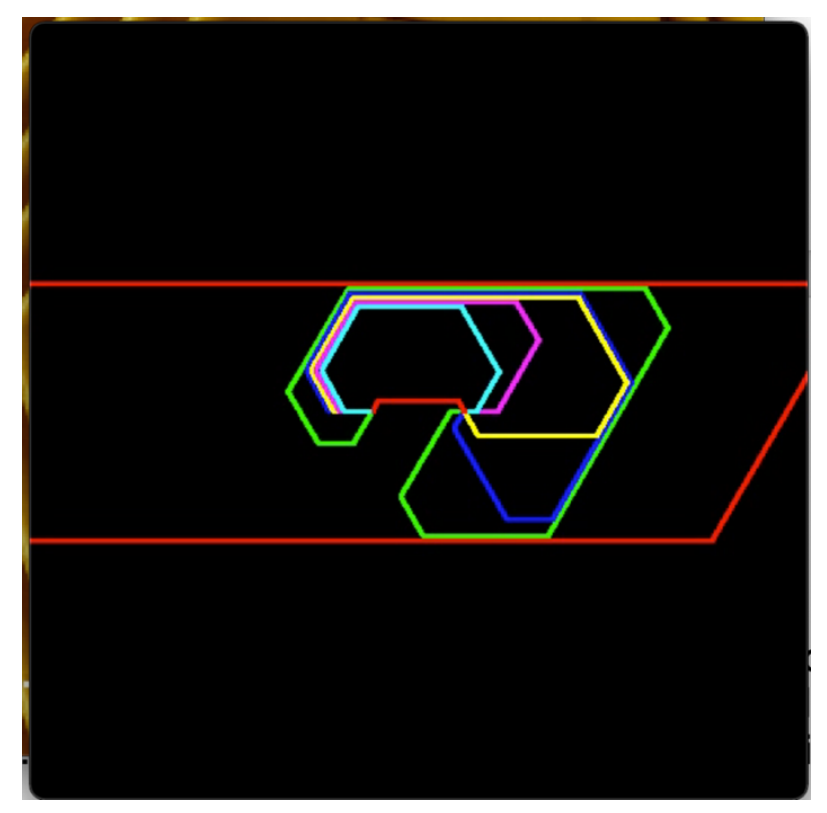

Video S5. Simulation of the formation of closed-loops on the L-cystine (0001) surface generated by a Frank dislocation comprising a closely spaced pair of heterochiral dislocations, using step velocity data obtained from the AFM measurements of the step velocities along the six different directions in a single elementary $c / 6$ layer. The six different colors correspond to the six different elementary layers in the unit cell along the $c$-axis. Reproduced with permission from reference 1. Copyright 2013 United States National Academy of Sciences.

\section{References}

1. Shtukenberg A. G.; Zhu, Z.; An Z.; Bhandari, M.; Song, P.; Kahr, B.; Ward, M. D. Illusory spirals and loops in crystal growth. Proc. Natl. Acad. Sci. 2013, 110, 17195-17198.

2. Rimer, J. D.; An, Z.; Zhu Z.; Lee, M. H.; Goldfarb, D. S.; Wesson, J. A.; Ward, M. D. Crystal Growth Inhibitors for the Prevention of L-Cystine Kidney Stones through Molecular Design. Science 2010, 330, 337-341. 